\title{
Mixture survival models methodology: an application to cancer immunotherapy assessment in clinical trials
}

\section{Lizet Sanchez ( $\square$ lsanchez@cim.sld.cu )}

Center of Molecular Immunology https://orcid.org/0000-0001-7747-1052

\section{Patricia Lorenzo-Luaces}

Center of Molecular Immunology

\section{Claudia Fonte}

University of Havana

\section{Agustin Lage}

Center of Molecular Immunology

\section{Research article}

Keywords: Survival mixture parametric models, long-term survival, lung cancer, immunotherapy

Posted Date: December 6th, 2019

DOI: https://doi.org/10.21203/rs.2.18321/v1

License: (c) (i) This work is licensed under a Creative Commons Attribution 4.0 International License.

Read Full License 
4 MIXTURE SURVIVAL MODELS METHODOLOGY: AN APPLICATION TO 5 CANCER IMMUNOTHERAPY ASSESSMENT IN CLINICAL TRIALS

6

$7 \quad$ Lizet Sánchez $^{1 \star}$, Patricia Lorenzo-Luaces ${ }^{1}$, Claudia Fonte $^{2}$, Agustin Lage $^{1}$

8

9

$10{ }^{1}$ Clinical Research Division, Center of Molecular Immunology, Calle 216 esq 15.

11 Atabey, Havana 11600, Cuba

$12{ }^{2}$ Faculty of Mathematics and Computing. University of Havana, San Lazaro y L,

13 Havana, Cuba

14

$15{ }^{\star}$ Corresponding author:

16 email: Isanchez@cim.sld.cu 


\section{Abstract}

18 Progress in immunotherapy revolutionized the treatment landscape for advanced

19 lung cancer, raising survival expectations beyond those that were historically anticipated with this disease. In the present study, we describe the methods for

21 the adjustment of mixture parametric models of two populations for survival analysis in the presence of long survivors. A methodology is proposed in several five steps: first, it is proposed to use the multimodality test to decide the number of subpopulations to be considered in the model, second to adjust simple parametric survival models and mixture distribution models, to estimate the parameters and to select the best model fitted the data, finally, to test the hypotheses to compare the effectiveness of immunotherapies in the context of randomized clinical trials. The methodology is illustrated with data from a clinical trial that evaluates the effectiveness of the therapeutic vaccine CIMAvaxEGF vs the best supportive care for the treatment of advanced lung cancer. The mixture survival model allows estimating the presence of a subpopulation of long survivors that is $44 \%$ for vaccinated patients. The differences between the treated and control group were significant in both subpopulations (population of shortterm survival: $p=0.001$, the population of long-term survival: $p=0.0002$ ). For cancer therapies, where a proportion of patients achieves long-term control of the disease, the heterogeneity of the population must be taken into account. Mixture parametric models may be more suitable to detect the effectiveness of immunotherapies compared to standard models.

39 KEY WORDS: Survival mixture parametric models, long-term survival, lung cancer, immunotherapy. 


\section{Introduction}

43 Advances in immunotherapy have revolutionized the treatment landscape for advanced lung cancer, raising survival expectations beyond those historically anticipated with this disease [1, 2]. More important than the improvements in the average survival time is the presence of a long and stable plateau with a heavy censoring at the tail of the curve [3]. This means that a proportion of patients are still alive even after long follow-up, it suggests the disease is controlled in a subgroup of long-term survivors. This new phenomenon is not currently captured by the most commonly used statistical procedures for the survival analysis, which generally assume that all patients are equally susceptible to the event of interest [3]. As an alternative, mixture parametric survival models have been proposed to take into account the heterogeneous structure in the data analysis $[4,5]$.

54 The finite mixture models have stayed widely used in many disciplines. For example, Nemec \& Nemec [6] apply the mixture models of astronomy distributions to study the number of distinct stellar populations in the Milky Way; Cameron and Heckman [7] use these types of models to assess the effect of family history on educational performance; Kollu [8] refers to several applications in engineering, Neale [9] in genetics and Land [10] in the social sciences. These models can be easily applied to the data set in which two or more subpopulations are mixed. Because of its flexibility, these models have been received intense attention in recent years, both from a practical and theoretical point of view. A complete description of the theory of mixture distributions and their applications can be found in McLachlan and Peel [11] or in Titterington et al. [12]. Finite mixture models have considered different distributions according to the data and problems that are modeled. For example, West [13] and Richardson and Green 
67 [14] studied the fit and applications of the mixture of normal distributions,

68 McLachlan and Peel [11] the mixture of t- students, Wiper and Rugginieri Gamma

69 distributions [15], and Tsionas Weibull [16]. However, in the analysis of data from

70 clinical trials, specifically those that evaluate the effect on survival is rarely used $71[17,18]$.

72 The present study proposes a methodology for the evaluation of the effect of 73 therapies in the presence of long-term survivors in the context of clinical trials.

74 We have structured the methodology as follows: in session 2.1 we describe the 75 multimodality test, a necessary condition for the application of the methods 76 proposed below; Session 2.2 describes the parametric models of simple survival 77 and mixture distributions; in session 2.3 the methods for estimating parameters 78 of the proposed models are explained; session 2.4 explains how to make the selection of the best model and finally in session 2.5 the hypothesis tests for comparing the effectiveness of therapies in the context of randomized clinical

81 trials are detailed. Additionally, the proposed methodology is illustrated for the 82 analysis of data from a clinical trial that evaluates the effectiveness of the 83 therapeutic vaccine CIMAvaxEGF for the treatment of advanced lung cancer.

\section{Methodology}

\subsection{Multimodality test}

Before adjusting a mixture parametric model, we must prove the existence of multimodality in the data, which justifies the application of the model. In this work, we assume the approach proposed by Silverman [19] and adapted by Hall and

89 York [20] for the case in which we want to test whether the true distribution of a variable in a population is unimodal or bi-modal. Formally, given a sample of a 
91 random variable with density function $f$ (in our case the survival time), denoting

92 by $\mathrm{j}$ the number of modes in $\mathrm{f}$, the hypothesis to test will be considered:

$93 \mathrm{HO}: \mathrm{j} \leq \mathrm{k}$, against $\mathrm{H} 1 \mathrm{:} \mathrm{j}>\mathrm{k}$,

94 Where $\mathrm{k} \in \mathrm{Z}+$ is the number of modes to contrast.

95 The test proposed by Silverman is based on the concept of a critical width. The 96 critical width will be the smallest width $\mathrm{h}$ for which it is verified that the estimate

97 of the density function has at most $\mathrm{k}$ modes. From this critical width, denoted by

$98 h_{k}$, Silverman proposes to use this parameter as a contrast statistic. Therefore

99 the null hypothesis will be rejected when $h_{k}$ is very "large", since this would mean

100 that it is necessary to overstate the core estimate for get a k - modal structure.

101 The critical width, $h_{k}$, would be the last value of $h$ before one of the modes

102 "separates" giving rise to an estimate with $(k+1)$ modes.

103 The $\mathrm{R}$ silvermantest package (available at http://cran.r-project.org/) has

104 implemented this method, which facilitates its implementation in practice.

\section{$105 \quad 2.2$ Survival parametric models}

\section{Simple model}

107 Survival analysis is a branch of statistics for analyzing the expected duration of

108 time until one or more events happen. For the subjects in which the event could

109 not be observed at the end of the follow-up period, the final state of the patient is

110 called "censored", since the actual duration of survival time cannot be known [21].

111 We define the random variable $\mathrm{T}$ as the time that elapses since the patient is

112 diagnosed with the disease until he dies. The survival function is defined as $\mathrm{S}(\mathrm{T})$

$113=\mathrm{P}(\mathrm{T}>\mathrm{t})$ where $0<\mathrm{t}<1$, which can be obtained as:

$114 S(t)=\int_{t}^{\infty} f(u) d u$ 
115 Where $f(u)$ is the density function. Simple models assume a unimodal distribution

116 of survival. The survival function, in this case, can be estimated from the data

117 assuming a parametric model. The most used are Exponential, Weibull, Log-

118 logistic and Lognormal (Table 1).

119 Table 1. Density and survival functions for continuous parametric distributions:

120 Exponential, Weibull, Log-logistic and Lognormal.

121

\begin{tabular}{lll}
\hline Model & $\begin{array}{c}\text { Density } \\
\text { function } \\
f(t)\end{array}$ & $\begin{array}{c}\text { Survival } \\
\text { function } \\
S(t)\end{array}$ \\
\hline Exponential & $\lambda \cdot e^{-\lambda t}$ & $e^{-\lambda t}$ \\
Weibull & $k \lambda t^{k-1} e^{-\lambda t^{k}}$ & $e^{-\lambda t^{k}}$ \\
Log-logistic & $\frac{k t^{k-1} \lambda}{\left(1+\lambda t^{k}\right)^{2}}$ & $\frac{1}{1+\lambda t^{k}}$ \\
Lognormal & $\frac{1}{t \sqrt{2 \pi} \sigma} e^{-\frac{(\log t-\mu)^{2}}{2 \sigma^{2}}}$ & $1-\Phi\left(\frac{\log t-\mu}{\sigma}\right)$
\end{tabular}

122

123

124

Mixture parametric survival model

125 A model with a mixture of distributions assumes the existence of two or more

126 populations within the sample, for each of which, the random variable $T$ follows a

127 different distribution. This means that there is heterogeneity in patient survival

128 and that individuals in each subpopulation have different risks of dying. In this

129 study, we will work with two-component mixture models [18].

130 The density function for this model is given by:

$131 \quad f(x ; \psi)=\pi_{1} f_{1}\left(x ; \theta_{1}\right)+\pi_{2} f_{2}\left(x ; \theta_{2}\right)$ 
132 Where $f_{1}$ and $f_{2}$ are the density functions of two of the distributions given in table

133 1. The parameter $\pi$ (population weight parameter) is such that $0<\pi \leq 1$, $\pi 1$ can

134 be interpreted as the proportion of one component or population in the model, 135 and $\pi_{2}=1-\pi_{1}$ the proportion of the other. The vector $\psi$ includes the vectors $\pi$;

$136 \theta 1$ and $\theta 2$.

$137 \quad 2.3$ Methods for parameter estimations

138 To estimate the parameters of the distribution of a simple model, the maximum

139 likelihood method implemented by the Optimum function of $\mathrm{R}$ was used. In the

140 mixture models, the parameters were estimated by this same method, using the

141 EM (Expectation-Maximization) algorithm, which was implemented in $\mathrm{R}$ and it will

142 be described later.

\section{Likelihood function}

144 Let $x 1, x 2, \ldots, x n$ be a countable set of values for the discrete random variable $X$ 145 and $\mathrm{P} \Psi(\mathrm{x})=\mathrm{P} \Psi(\mathrm{X}=\mathrm{x})$. For the correct values of the parameter vector $\Psi$ the 146 function $P$ allows us to find the probability that $X$ takes each of the values $x 1$, $147 \times 2, \ldots$ Let's look at this same function from another point of view: let's say we 148 know that the random variable $\mathrm{X}$ takes the given values and follows a distribution $149 \mathrm{P} \psi(x)$ for an unknown value of $\psi$, so let $\Phi$ be the space of the possible values

150 of $\psi$, we can interpret $\mathrm{P} \psi(\mathrm{x})$ as a function of $\Psi$ given $\mathrm{x}$. Seen in this way, the 151 function $\mathrm{P} \Psi(\mathrm{x})$ is known as the likelihood function and is denoted by $L x(\Psi)$.

152 In general, when you have a continuous random variable, for uncensored data, 153 the likelihood function is given by $\operatorname{Lx}(\psi)=\prod_{i=1}^{n} f(x i ; \psi)$, it is assumed that the 154 experiments in which the values of $X$ were found were independent and $f(x i ; \Psi)$ 155 (the density function corresponding to the distribution with which the variable is 
156 assumed) replaces $\mathrm{P} \Psi(\mathrm{x})$. Thus, if we considered the mixture distribution model

157 defined in the previous section, we obtain:

$\mathrm{Lx}(\Psi)=\prod_{i=1}^{n}(\pi 1 \mathrm{f} 1(\mathrm{xi} ; \theta 1)+\pi 2 \mathrm{f} 2(\mathrm{xi} ; \theta 2))$

159 A problem arises when considering censored data since the value of the variable

160 for all the elements of the sample is not known exactly. However, taking into

161 account the type of censorship existing in our data, this can be solved using the survival function instead of the density function (Since the information we have is that the individual survived at least a number of months, we take the probability

164 of surviving $y$ or more months instead of surviving exactly $y$ months). Thus, the

165 likelihood function is as follows:

166

$$
\operatorname{Lx}(\psi)=\prod_{i=1}^{n 1}(\pi 1 \mathrm{f} 1(\mathrm{xi} ; \theta 1)+\pi 2 \mathrm{f} 2(\mathrm{xi} ; \theta 2)) \prod_{j=1}^{n 2}(\pi 1 \mathrm{~S} 1(\mathrm{xj} ; \theta 1)+\pi 2 \mathrm{~S} 2(\mathrm{xj} ; \theta 2))
$$

Where $n_{1}$ and $n_{2}$ are the number of not censored and censored individuals

169 respectively.

170 Given a space of possible values $\theta$ for the parameter $\psi$, the maximum likelihood method consists of finding the maximum likelihood estimate $\psi \hat{\imath}=\arg \max \psi \in \Phi L x$ $(\Psi)$. Taking into account the monotony of the logarithm function, it is sometimes convenient to use $\operatorname{Lx}(\psi)=\ln L x(\psi)$ (log-likelihood function) instead of the

174 maximum likelihood function, to calculate the maximum likelihood estimate.

\section{EM algorithm}

176 We now have to answer the question: How to maximize the log-likelihood

177 function? For this, there are several methods among which are the Moments

178 Method, the EM Method, and the Fisher Information Method. More information

179 on these can be found in [22]. In this study, we will use the EM algorithm, which

180 is one of the most used in the literature. 
181 To make the notation less cumbersome while describing the algorithm, it is 182 assumed that the data is not censored, otherwise they would have, $x 1, x 2, \ldots$, $183 \mathrm{xn} 1$ uncensored data and $\mathrm{y} 1, \mathrm{y} 2, \ldots$, and $\mathrm{n} 2$ censored data, and the density or 184 survival function would be taken as appropriate, the rest being analogous.

185 Let $x 1, x 2, \ldots, x n$ the values that the variable takes in a sample of individuals.

186 The information can be considered incomplete since it is unknown to which of the 187 two existing populations, in which the random variable distributes differently, 188 within the sample each individual belongs. To complete the information, the 189 indicator variable $z i, i=1,2, \ldots, n$ is taken, where $z i=1$ if $x 1$ is given by $f 1$ and $190 \mathrm{zi}=0$ otherwise. The system (zi, xi) will contain the complete information of the 191 data. Initially, the zi values can be found as a random sample of Bernoulli 192 distribution $(\alpha)$, where $\alpha$ is the weight parameter.

193 The EM algorithm is divided into two stages, stage $E$ and stage $M$. Each of these 194 is described below in the $k+1$-th iteration. In stage $E$, the value of zi is estimated 195 from the conditional expectation $\mathrm{E}$ (zi | xi), which, using the Bayes total probability 196 formula, can be found as:

$$
\hat{Z}_{i}=E\left(\hat{Z}_{i} \mid X_{i}\right)=\frac{\pi 1 f 1\left(x i, \theta 1^{(k)}\right)}{\pi 1 f 1\left(x i, \theta 1^{(k)}\right)+\pi 2 f 2\left(x i, \theta 2^{(k)}\right)}
$$

198 Where $\theta 1(\mathrm{k})$ and $\theta 2(\mathrm{k})$ are the parametric vectors of the distributions of each 199 component, estimated in the k-th iteration.

200 In step $M$, the parameter values are maximized, completing the information with 201 the ẑi found above.

$202 \quad \Pi^{(\mathrm{k}+1)}=\frac{1}{n} \sum_{i=1}^{n} \hat{z}_{i}$

$203 \quad \theta_{1}{ }^{(\mathrm{k}+1)}=\arg \max \theta 1 \in \Phi \sum_{i=1}^{n} \hat{z}_{i} \ln (f 1(x i, \theta 1))$

$$
\theta_{2}{ }^{(\mathrm{k}+1)}=\arg \max \theta 2 \in \Phi \sum_{i=1}^{n} \hat{z}_{i} \ln (f 1(x i, \theta 2))
$$


205 For a single component, knowing the individuals belonging to that component, 206 the problem of finding the values of the parameters that maximize the likelihood

207 function is no more difficult than estimating the parameters of a simple model.

208 This was done, using the Optimum function of R.

209 The iterations of stages $\mathrm{E}$ and $\mathrm{M}$ of the algorithm are repeated until $\mid \mathrm{Ix}(\Psi(\mathrm{k}+$

$2101))-\operatorname{Ix}(\Psi(k)) \mid$ is less than a small value, specified previously. The EM algorithm

211 satisfies that $\operatorname{lx}(\Psi(k+1)) \geq \operatorname{lx}(\Psi(k))$, this property is the fundamental reason for

212 its convergence [21].

2132.4 Method for selecting the best model

214 If the actual distribution $(G)$ of the variable with density function $g$ is known, to

215 measure how our model $f\left(x \mid \theta^{\wedge}\right)$ approximates, the Kullback-Leibler Information

$216(\mathrm{~K}-\mathrm{L})$ is used. $\mathrm{K}-\mathrm{L}$ is given by:

$$
E_{G(X)}\left[\ln \frac{g(X)}{f(x, \theta)}\right]=E_{G(X)}[\ln g(X)]-E_{G(X)} \ln f\left(x, \theta^{\wedge}\right)
$$

218 Where the best model will be the one that differs to a lesser extent from the real

219 one and, therefore, the value of the K-L Information is lower. Moreover, in most cases, as in ours, the actual distribution of the variable is unknown. However, note that the expression $E G(X)[\ln g(X)]$ is a common constant for all models, so that the best model will be that, such that the value of $E G(X) \ln f\left(x, \theta^{\wedge}\right)$ be higher. This value can be approximated by the log-likelihood function plus a bias $b\left(\theta^{\wedge}\right)$. Depending on how this approach is taken, several Information Criteria are defined for the selection of the best model. This paper uses the Akaike Information Criterion (AIC), defined as $-2 \ln \left(\operatorname{Lx}\left(\theta^{\wedge}\right)\right)+2 \mathrm{k}$.

\subsection{Hypothesis testing to evaluate the effect of the treatment}

228 The Log-rank test is the most used method to compare the survival of groups. It

229 has as a null hypothesis that there are no differences between the populations 
230 for the occurrence of an event at any time of the follow-up. The analysis is based

231 on the time of the events (i.e. deaths) of each group, which is compared with the

232 expected number of events if there were no differences between the groups. In

233 this work, we have used this test for the direct comparison of the Treated and

234 Control groups for the case in which the existence of a single homogeneous

235 population is assumed, that is, when a simple survival model is assumed (model $2360)$.

237 For the case in which we assume the existence of two subpopulations (model 1),

238 we have taken, as a cut-off point, the intersection of the estimated density

239 functions for each subpopulation. All patients with survival lower of this cut-off

240 point have been classified in the short-term population and all patients above the

241 cut-off are considered to belong to the long-term population.

242 A new immunotherapy (IT) treatment being evaluated may not have an effect on

243 the parameters for both populations, or it could modify the proportion of patients,

244 or the median survival of some subpopulation, or simultaneously both effects [23].

245 To evaluate the effect of immunotherapy we allowed the model parameters to

246 depend on the treatment. Table 2 summarizes the assumptions of the model and

247 hypothesis tests considered on the parameters to assess the effect of IT.

248 Table 2: Hypothesis tested to evaluate the effect of the treatment in the mixture 249 parametric survival model

\begin{tabular}{|c|c|c|c|c|}
\hline Variant $^{\mathrm{A}}$ & Hypothesis & Explanation & Mean structure & $\begin{array}{l}\text { Mixing } \\
\text { proportions }\end{array}$ \\
\hline 2 & $\begin{array}{l}\mathrm{H} 0: \beta 1 \mathrm{k}=0, \\
\mathrm{k}=1,2\end{array}$ & $\begin{array}{l}\text { There is an effect of the } \\
\text { therapy on the parameters } \\
\text { for median overall survival, } \\
\text { but not on the mixing } \\
\text { proportion parameters. }\end{array}$ & $\begin{array}{l}\lambda \mathrm{k}=\quad \beta 0 \mathrm{k}+ \\
\beta 1 \mathrm{k}^{*} \mid \mathrm{T} 1, \\
\mathrm{k}=1,2\end{array}$ & $\begin{array}{l}\pi 1=\operatorname{logit}(z 0), \\
\pi 2=1-\pi 1\end{array}$ \\
\hline
\end{tabular}




\begin{tabular}{|c|c|c|c|c|}
\hline 3 & $\begin{array}{l}\mathrm{H} 0: \alpha=0 \\
\mathrm{H} 0: z 1=0\end{array}$ & $\begin{array}{l}\text { There is an effect of the } \\
\text { therapy on the mixing } \\
\text { proportion parameters, but } \\
\text { not on the parameters for } \\
\text { median overall survival. }\end{array}$ & $\lambda 2=\lambda 1+\alpha$ & $\begin{array}{l}\pi 1=\operatorname{logit}(z 0+ \\
\left.z 1^{*} \mid T\right) \\
\pi 2=1-\pi 1\end{array}$ \\
\hline 4 & $\begin{array}{l}\mathrm{H} 0: \beta 1 \mathrm{k}=0 \\
\mathrm{HO}: z 1=0\end{array}$ & $\begin{array}{l}\text { There is an effect of the } \\
\text { therapy on the mixing } \\
\text { proportion parameters and } \\
\text { on the parameters for } \\
\text { median overall survival. }\end{array}$ & $\begin{array}{l}\lambda 1 \mathrm{k}=\quad \beta 0 \mathrm{k}+ \\
\beta 1 \mathrm{k}^{*} \mathrm{IT} 1 \\
\mathrm{k}=1,2\end{array}$ & $\begin{array}{l}\pi 1=\operatorname{logit}(z 0+ \\
\left.z 1^{*} \mid T\right) \\
\pi 2=1-\pi 1\end{array}$ \\
\hline
\end{tabular}

250 A To incorporate the effect of treatment in this model, we considered three variants

251 Definition of terms:

$252 \mathrm{H}_{0}$, the null hypothesis

253 IT is the variable for the treatment groups (Control: $I T=0$ and CIMAvaxEGF: IT $T_{1=1}$ )

$254 \mathrm{k}=1,2$ represent the short- and long-term survival populations

$255 \pi_{1}$ and $\pi_{2}=$ the proportion of short-term and long-term survivors, respectively;

256 (where $k=1$ or 2 ), with the restriction that $0<\pi_{k} \leq 1$ and $\pi_{1}+\pi_{2}=1$, are the mixing

257 proportions for the kth population.

$258 \lambda_{k}$ is the scale parameters for the Weibull distribution

$259{ }^{*}$ Note that if any of the $\beta 1 \mathrm{k}$ is not significant, the average overall survival for

260 subpopulation $\mathrm{k}$ would not depend on the treatment and would be similar for 261 treaties and controls.

\section{Application to the data}

\subsection{Data description}

265 Data from a randomized, multicenter, controlled clinical trial in patients with 266 advanced NSCLC was used. The phase III trial evaluated the efficacy of

267 CIMAvaxEGF, an EGF-based cancer vaccine compared with best supportive

268 care (Control). The recruitment period was between July 5, 2006, and January 3,

269 2012. Patients in advanced stages (IIlb / IV) who received 4 to 6 cycles of

270 platinum-based first-line chemotherapy and who had confirmation were included.

271 of stable disease or an objective response at least 4 weeks before randomization. 
272 A total of 405 patients were randomized (2: 1) to vaccinated or control groups.

273 The vaccination group $(n=270)$ received the CIMAvaxEGF vaccine plus the best

274 supportive care while the control group $(n=135)$ only received the best

275 supportive therapy. Both groups were balanced according to the prognostic

276 factors of the disease. More details can be found in [24]. The main evaluation

277 criterion of the trial was overall survival (OS).

$278 \quad 3.2$ Existence of bimodality

279 The $p$-values obtained for the different $k$ considered in the Silverman test are

280 shown in Fig 1. It can be seen that the unimodality hypothesis is rejected for both

281 treaties $(p=0.01)$ and controls $(p<0.001)$. The first value of the test that is not

282 significant is $\mathrm{k}=2$ in both cases, which indicates that survival in the two groups

283 has a bimodal distribution.

Fig 1. $P$ values according to the different $k$ considered in the Silverman multimodality test for the survival distribution of the control groups and treated with CIMAvaxEGF. For $k=1$ the values of $p$ are less than 0.05 , so the null hypothesis that the distribution is unimodal is rejected. The first non-significant value of $p$ is obtained in both cases when $k=2$ which is interpreted in this case as that the sample is bimodal.

\subsection{Models Fitting and best model selection}

293 For the illustration of the proposed methodology, we have considered a Weibull distribution or a mixture of Weibull distributions. Table 3 shows the results of the adjustment of the different models and the estimated values of the median survival time and the proportions for the subpopulations. The two-component 
297 model resulted in a much better fit than the model assuming a homogeneous

298 population (AIC: 2452 vs. 2783). The best fit is achieved with model 4 ( $\mathrm{AIC}=$

299 2448). This is the survival model that assumes the mixture of two distributions

300 and that both parameters, median survival, and mixing proportion, depend on

301 treatment. The components represent short-term and long-term survival

302 subpopulations. A significant increase of $10 \%$ of patients with long-term survival

303 is observed in the group treated with CIMAvaxEGF with respect to the control

$304 \operatorname{group}(p=0.005)$.

305 Table 3: Adjustment and estimates of model parameters assuming different

306 hypotheses.

\begin{tabular}{|c|c|c|c|c|c|c|}
\hline Model & $\mathrm{AIC}$ & groups & Median1 & Median2 & $\pi 1$ & $\pi 2$ \\
\hline 0 (1 component) & 2783 & - & 9.21 & & & \\
\hline 1 (2 components) & 2452 & - & 7.28 & 27.29 & 0.55 & 0.45 \\
\hline \multirow[t]{2}{*}{2 (m Treatment) } & 2449 & Control & 6.28 & 26.24 & \multirow[t]{2}{*}{0.62} & \multirow[t]{2}{*}{0.37} \\
\hline & & Treated & 8.35 & 34.95 & & \\
\hline \multirow[t]{2}{*}{3 ( $\pi \sim$ Treatment) } & 2449 & Control & \multirow[t]{2}{*}{7.27} & \multirow[t]{2}{*}{27.25} & 0.68 & 0.32 \\
\hline & & Treated & & & 0.50 & 0.50 \\
\hline \multirow[t]{2}{*}{$4(\mathrm{~m}, \pi \sim$ Treatment) } & 2448 & Control & 6.69 & 26.01 & 0.66 & 0.34 \\
\hline & & Treated & 7.91 & 30.78 & 0.56 & 0.44 \\
\hline
\end{tabular}

307

$308 \quad 3.4$ Application to the evaluation of the effect of therapies

309 Fig 2 shows the survival curves for the short and long survival populations for

310 both groups considering the best fit model (Model 4). The differences between 
311 the treated and control groups were significant in both subpopulations (short-lived

312 population: $p=0.028$; long-lived population: $p=0.0001)$.

314 Fig 2: Survival curves for short and long survival populations

\section{Discussion}

317 In this work, a useful methodology was proposed to take into account the

318 heterogeneity in the survival data. The methodology was illustrated in the

319 evaluation of the effect of a new immunotherapy. Specifically, for the study of the

320 survival of lung cancer patients in advanced stages, it is shown that the use of

321 mixture distribution models are more appropriate than the use of simple models.

322 The results of the study can be interpreted from three points of view: the existence

323 of subgroups with different risks of dying within the population of patients with

324 advanced lung cancer regardless of the therapy used; the effects of the

325 therapeutic vaccine CIMAvaxEGF in the subgroups of patients identified as short

326 and long survival; and the adaptation of current statistical methods to face the

327 transition from advanced cancer to a chronic disease.

328 It has been shown that therapies for certain types of cancer induce a subset of 329 long-term survivors, such as melanoma [25], breast cancer [26] and multiple

330 myeloma [27]. Specifically for advanced lung cancer, evidence has been found

331 of patients at different risk of death in both population studies [29-30] and in 332 clinical trials [31-33]. The identification of this finding before modern therapies 333 suggests that some patients experience prolonged survival regardless of 334 treatment [29]. In this location, the fraction of long survival has been estimated in 335 about half of the participants in the clinical trial, while in population studies it is 
336 estimated at around 10\% [30]. This difference is attributed to the restrictive 337 selection criteria in clinical trials, which means that there is an overrepresentation 338 of patients more likely to be long-term survivors.

339 It should be noted that in many of the studies that refer to the presence of long 340 survivors, traditional survival methods that assume homogeneity in the sample 341 are used, a stratified analysis is done according to groups defined by biomarkers 342 or the group of patients is divided arbitrarily into of short and long survival. The 343 biological characteristics that define the heterogeneity of the data or the patient 344 populations that will evolve to a short or long survival are not always known in 345 advance. Biomarkers are often not incorporated into the design of the clinical trial 346 but arise in secondary analyzes that are performed afterward. The definition of 347 long survivors varies widely for each cancer location and among studies reported 348 in the literature. To more accurately measure the clinical benefits of therapies, 349 the American Society of Clinical Oncology and the European Society of Medical 350 Oncology have proposed the ASCO-VF and ESMO-MCBS scales respectively.

351 Both proposals were recently amended to incorporate bonuses and adjustments 352 that capture the tail of the survival curve $[34,35]$. However, a study that critically analyzes the application of these proposals in 107 clinical trials concludes that neither of the two proposals was consistent as a measure of the absolute survival benefit [36].

356 The models of the mixture of distributions presented here are an alternative to 357 consider and can be a useful tool for reinterpreting data from clinical trials already carried out based on taking into account heterogeneity. In our study, we do a retrospective re-analysis of the data from a clinical trial, and certainly, the

360 percentage of long survivors may be overestimated. However, due to the 
361 randomization process, this must have occurred to the same extent in the treated

362 and control groups. More important than the estimation itself of the fraction of

363 long survivors, is the proposal to apply the mixture distribution model to test

364 hypotheses about the effect of immunotherapies in clinical trials. This is in our

365 opinion the greatest novelty of this work.

366 The methodology proposed here has the advantage that heterogeneity assumes

367 without requiring groups to be defined beforehand. This is illustrated for the case

368 in which there are two populations, but can easily be extended to the case where

369 there are multiple subpopulations. It is important to keep in mind that the

370 estimation of the mixture fraction can be very sensitive to the parametric

371 distribution chosen to work [28]. For this reason, the selection of the parametric

372 distribution to model the observed data should be done carefully. McCullagh and

373 Barry [37] proposed a model selection process algorithm and recommended

374 adjusting different distributions to the data and selecting the best distribution

375 using one of the available information criteria.

376 Once the existence of heterogeneity in the main variable that is evaluated in

377 retrospective exploratory studies has been detected, confirmation of this finding

378 is imposed through the design of new clinical trials. This is one of the current

379 challenges facing statisticians working in immuno-oncology. Even more so when

380 the implementation of sequential Bayesian designs with intermediate analyzes

381 has become a standard practice in phase III clinical trials to allow early

382 termination of the studies. The software available for sample size calculation, 383 such as PROC POWER in SAS, the survival analysis module in PASS, the 384 powerSurvEpi package in $\mathrm{R}$, is based on standard proportional hazard $(\mathrm{PH})$ 385 models that are not appropriate for the design in the presence of a fraction of 
patients with long survival. Cai [38] has proposed a methodology, which he has

387 applied and implemented in the NPHMC R package. In his work, he establishes

388 the calculation of sample size in the case in which a group of patients reaches a

389 cure with the treatment. However, in the case of clinical trials in patients in

390 advanced stages not operated, patients are not cured, but the new treatments

391 are focused on achieving stabilization of the disease. New studies could address

392 the proposal of clinical trial designs that take into account the mix of different

393 distributions or risks in the subpopulations of the study.

394 The implementation in the exposed case study confirmed that vaccination with

395 CIMAvaxEGF prolongs the survival of patients with advanced lung cancer.

396 Although in both populations, short and long survival, patients are benefited with

397 immunotherapy, the benefit is markedly greater in the long-lived population.

398 Subsequent studies could propose the characterization and identification of

399 biomarkers predictive of successful treatment with CIMAvaxEGF of both

400 subpopulations or the selection of patients with a high benefit with this

401 immunotherapy.

402 Future research could focus on the reproducibility of the findings of the effect of 403 other treatments for lung cancer on the mixture fraction or on the survival of 404 subpopulations. Alternatively, the prospective identification of patients with long405 term survival potential may allow the validation of biomarkers predictive of the 406 effect of therapies and favor the use of more personalized therapeutic 407 approaches. The phenomenon of multimodality and the effect of 408 immunotherapies on other types of tumors could also be studied. Studies are 409 needed to determine the host factors and molecular characteristics that influence 410 the heterogeneity of the survival of cancer patients. 
412 Acknowledgement

413 We thank all the patients and staff of all the institutions that were involved in the

414 clinical trial.

\section{References}

416 1. Massarelli E, Papadimitrakopoulou V, Welsh J, Tang C, Tsao AS.

417 Immunotherapy in lung cancer. Translational lung cancer research. $418 \quad 2014 ; 3(1): 53-63$.

419 2. Carrizosa DR, Gold KA. New strategies in immunotherapy for non-small cell 420 lung cancer. Translational lung cancer research. 2015;4(5):553- 9.

3. Chen TT. Statistical issues and challenges in immunooncology. J Immuno

422 Ther Cancer. $2013 ; 1: 18$.

423 4. McLachlan GJ, Peel D. Finite Mixture Distributions. New York: Wiley; 2000.

424 5. Titterington DM, Smith AFM, Makov UE. Statistical Analysis of Finite Mixture $425 \quad$ Distributions. New York: Wiley;1985.

426 6. Nemec L, Nemec AFL. Mixture models for studying stellar populations. I. 427 Univariate mixture models, parameter estimation, and the number of discrete 428 population components. Publications of the Astronomical Society of the $429 \quad$ Pacific. 1991; 103:95-121.

430 7. Cameron SV, Heckman JJ. Life cycle schooling and dynamic selection bias: 431 Models and evidence for five cohorts of American males. Journal of Political 432 Economy. 1998;106,262-333.

433 8. Kollu R, Rayapudi SR, Narasimham SVL and Pakkurthi KM. Mixture probability 434 distribution functions to model wind speed distributions. International Journal 435 of Energy and Environmental Engineering. 2012 3:27. 
436 9. Neale MC. A Finite Mixture Distribution Model for Data Collected from Twins.

437 Twin Research. 2003;6(3):235-239.

438 10. Land KC. Introduction to the Special Issue on Finite Mixture Models.

439 Sociological Methods \& Research. 2001;29(3):275-281.

440 11. McLachlan G, Peel D. Finite Mixture Models. Jonh Wiley \& Sons, Inc. 2000

441 12. Titterington, DM, Smith, AFM and Makov, UE. Statistical Analysis of Finite $442 \quad$ Mixture Distributions. London: Wiley. 1985

443 13. West M. Approximating posterior distributions by mixtures. J. Roy. Statist. $444 \quad$ Soc. $1983 ; 55: 409-422$.

445 14. Richardson S, Green P. On Bayesian analysis of mixtures with an unknown 446 number of components. J. Roy. Statist. Soc. Ser. 1997;B59:731-792.

447 15. Wiper DR, Rugieri F. Mixture with gamma distribution with applications. $448 \quad J$ Journal of computational and graphical statistics. 2001;10(3):440-454.

449 16. Tsionas E. Bayesian analysis of finite mixtures of Weibull distributions. $450 \quad$ Communication in statistics. part A. 2002,31(1):37-48.

451 17. Erişoğlu U, Erişoğlu M, Erol H. A Mixture Model of Two Different Distributions 452 Approach to the Analysis of Heterogeneous Survival Data, International 453 Journal of Computational and Mathematical Sciences. 2011; 5:2.

454 18. Marin JM, Rodriguez-Bernal MT, Wiper MP: Using weibull mixture 455 distributions to model heterogeneous survival data. Communicat Stat. 2005, $456 \quad 34: 673-684$.

457 19. Silverman BW. Using kernel estimates to investigate multimodality, J. Royal $458 \quad$ Statist. Sot. B 1981;43:97-99.

459 20. Hall P, York M. On the calibration of Silverman's test for multimodality, $460 \quad$ Statistica Sinica.2001;11:515-36 
21. Collet D. Modelling Survival Data in Medical Studies, 2nd edition, Chapman \&Hall/CRC. 2003.

22. Wu CFJ. On the convergence properties of the EM alorighm. The Annals of Statistics. 1983;11:95-103

23. Sanchez L, Muchene L, Lorenzo-Luaces P, Viada C, Rodriguez PC, Alfonso S, et al. Differential Effects of Two Therapeutic Cancer Vaccines on Shortand Long-Term Survival Populations among Patients with Advanced Lung Cancer. Semin Oncol. 2018;45:52-57.

24. Rodriguez PC, Popa X, Martínez O, Mendoza S, Santiesteban E, Crespo T, et al. A Phase III Clinical Trial of the Epidermal Growth Factor Vaccine CIMAvax-EGF as Switch Maintenance Therapy in Advanced Non-Small Cell Lung Cancer Patients. Clinical Cancer Research. 2016;22(15):3782-90.

25. Eggermont AM, Suciu S, Testori A, Santinami M, Kruit WH, Marsden J, et al. Long-term results of the randomized phase III trial EORTC 18991 of adjuvant therapy with pegylated interferon alfa-2b versus observation in resected stage III melanoma. J Clin Oncol. 2012;30(31):3810-3818.

26. Ambs S: Prognostic significance of subtype classification for short- and longterm survival in breast cancer: survival time holds the key. PLoS Med 2010, 7(5):e1000281.

27. Othus M, Barlogie B, Leblanc ML, Crowley JJ: Cure models as a useful statistical tool for analyzing survival. Clin Cancer Res. 2012;18(14):37313736.

28. Sanchez L, Lorenzo-Luaces P, Viada C, Galan Y, Ballesteros J, Crombet T et al. Is there a subgroup of long-term evolution among patients with advanced 
lung cancer?: hints from the analysis of survival curves from cancer registry data. BMC cancer. 2014;14:933 - 9.

29. Angelis R, Capocaccia R, Hakulinen T, Soderman B, Verdecchia A. Mixture models for cancer survical analysis: aplication to population-based data with covariates. Stat Med. 1999;18:144-454.

30. Davis JS, Prophet E, Peng H-L, et al. Potential influence on clinical trials of long-term survivors of stage IV non-small-cell lung cancer. JNCI Cancer Spectr. 2019.

31. Giroux Leprieur E, Lavole A, Ruppert AM, Gounant V, Wislez M, Cadranel J, et al. Factors associated with long-term survival of patients with advanced non-small cell lung cancer. Respirology. 2012;17(1):134-42. 32.

32. Kaira K, Takahashi T, Murakami H, Tsuya A, Nakamura Y, Naito T et al. Longterm survivors of more than 5 years in advanced non-small cell lung cancer.

33. Satoh H, Ishikawa H, Ohara G, Kagohashi K, Kurishima K, Ohtsuka M et al. Long-term survivors after chemotherapy in advanced non-small cell lung cancer. Anticancer research. 2007;27(6C):4457-60.

34. Schnipper LE, Davidson NE,Wollins DS, et al. Updating the American Society of Clinical Oncology Value Framework: revisions and reflections in response to comments received. J Clin Oncol. 2016; 34(24):2925-2934.

35. Cherny NI, Dafni U, Bogaerts J, et al. ESMO-Magnitude of Clinical Benefit Scale version 1.1. Ann Oncol. 2017;28

36. Saluja R, Everest L, Cheng S, Cheung M, Chan KKW. Assessment of whether the American Society of Clinical Oncology's value framework and the European Society for Medical Oncology's magnitude of clinical benefit scale 

measure absolute or relative clinical survival benefit: a meta-analysis of

511 randomized clinical trials.JAMA Oncol. 2019;5(8):1188-1194.

512 37. McCullagh L, Barry M: Survival analysis used in company submissions to the 513 national centre for pharmacoeconomics. Ireland Value Health. 2013; 16:A398

514 38. Cai C, Zou Y, Peng Y, Zhang J. smcure: an R-package for estimating 515 semiparametric mixture cure models. Comput Methods Programs Biomed. $516 \quad 2012 ; 108(3): 1255-60$.

\section{Author Contributions}

519 Conceived and designed the study: LS and AL. Implemented the methodology

520 and analyzed the data: LS, PL and CF. Wrote the paper: LS, CF and AL. All authors participate in the interpretation of the data, critically revised subsequent drafts of the manuscript, and approved the final version.

\section{Availability of data}

524 The dataset supporting the conclusions of this article is included within the article 525 and its additional file.

\section{Ethics declarations}

\section{Ethics approval and consent to participate}

528 The trial was approved by local ethics review boards and the Cuban Regulatory

529 Agency. The trial was conducted in accordance with the principles of the

530 declaration of Helsinki and Good Clinical Practice guidelines. It was registered

531 at the Cuban Registry of Clinical Trials, a WHO-validated public registry

532 http://www.who.int/ictrp/network/rpcec/en, trial number RPCEC00000161)

533 Consent for publication 
534 This manuscript does not contain any details, images, or videos that might leed

535 to identification of an individual patient.

536 Competing interests

537 The authors declare that there are no conflicts of interest.

538

539 Financial Disclosure Statement

540 The authors received no specific funding for this work

541 


\section{Figures}
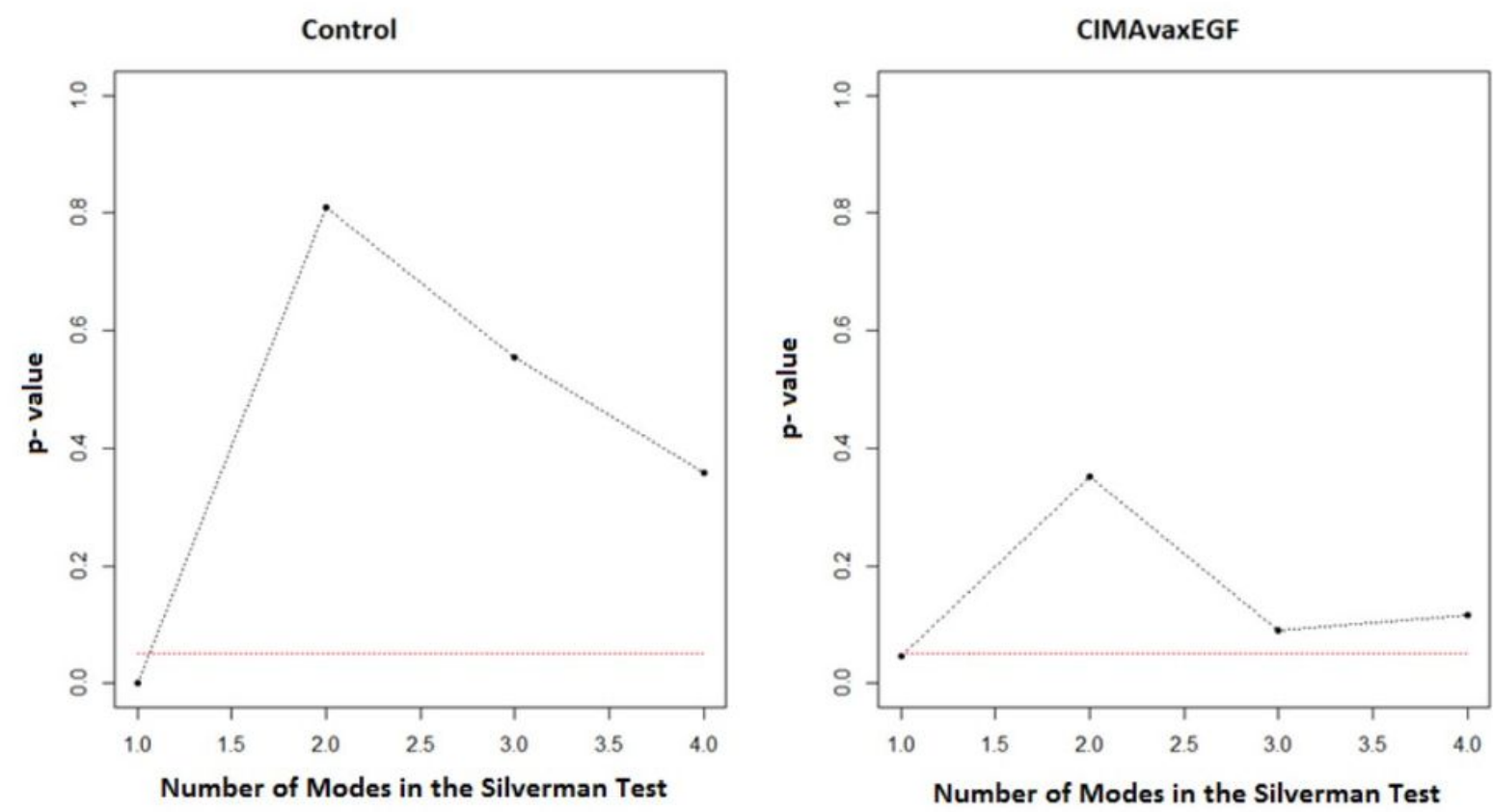

\section{Figure 1}

$P$ values according to the different $k$ considered in the Silverman multimodality test for the survival distribution of the control groups and treated with CIMAvaxEGF. For $k=1$ the values of $p$ are less than 0.05 , so the null hypothesis that the distribution is unimodal is rejected. The first non-significant value of $\mathrm{p}$ is obtained in both cases when $\mathrm{k}=2$ which is interpreted in this case as that the sample is bimodal. 


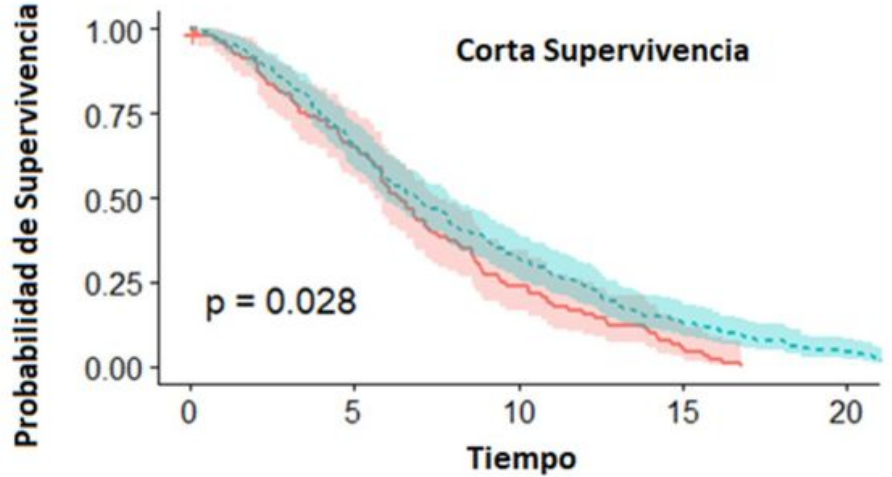

Número de pacientes en riesgo

\begin{tabular}{cccccc} 
Control & 93 & 60 & 22 & 5 & 0 \\
EGF 194 & 128 & 62 & 26 & 8 \\
\multicolumn{4}{c}{ Tratamiento } & + Control *- EGF
\end{tabular}

Figure 2

Survival curves for short and long survival populations

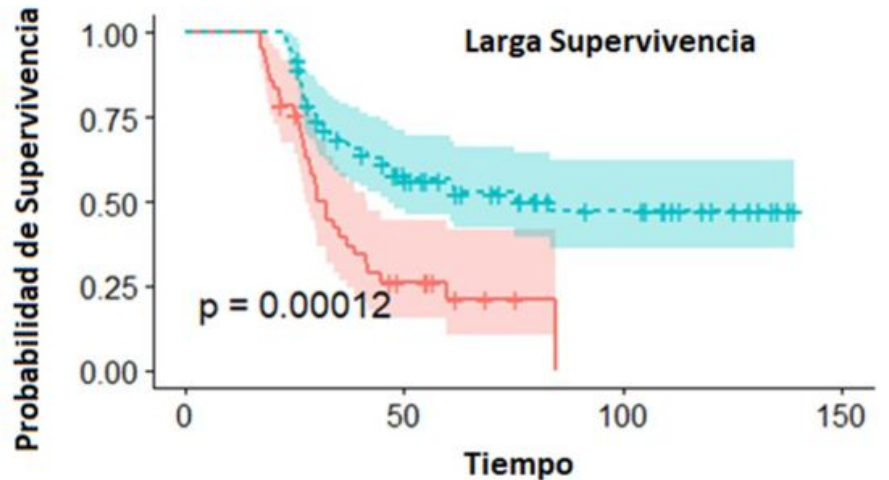

Número de pacientes en riesgo

$\begin{array}{cccc}\text { Control } & 42 & 8 & 0 \\ \text { EGF } & 76 & 35 & 15 \\ & & & \\ & & \text { Tratamiento } & + \text { Control }+*-\text { EGF }\end{array}$

